\title{
Optical Refractive Index of Massive Particles and Physical Meanings of Left-handed Media
}

\author{
Jian-Qi Shen ${ }^{1,2}$ * \\ ${ }^{1}$ Centre for Optical and Electromagnetic Research, State Key Laboratory of Modern Optical \\ Instrumentation, Zhejiang University, Hangzhou Yuquan 310027, P.R. China \\ ${ }^{2}$ Zhejiang Institute of Modern Physics and Department of Physics, Zhejiang University, \\ Hangzhou 310027, P.R. China
}

(November 9, 2018)

\begin{abstract}
In this Letter the expression for the refractive index of de Broglie wave in the presence of a potential field is obtained and based on this, the physical meanings of negative index of refraction is revealed. We demonstrate that the electromagnetic wave propagation in a left-handed medium with negative refractive index behaves just like that of antiphotons, which is required of the complex vector field theory. It is believed that the complex vector field theory is helpful in considering the wave propagation and photonic band gap structure in the left-handed medium photonic crystals with a periodicity in negative and positive index of refraction.
\end{abstract}

*E-mail address: jqshen@coer.zju.edu.cn 
PACS: 78.20.Ci; 42.70.-a; 03.50.-z; 42.25.-p

Keywords: Negative refractive index; Left-handed media; Complex vector field theory

Typeset using REVTEX 
More recently, a kind of artificial composite metamaterials (the so-called left-handed media) having a frequency band where the effective permittivity and the effective permeability are simultaneously negative attracts considerable attention of many authors both experimentally and theoretically $[1-6,10,11]$. In $1967^{1}$, Veselago first considered this peculiar medium and showed from Maxwellian equations that such media having negative simultaneously negative $\epsilon$ and $\mu$ exhibit a negative index of refraction, i.e., $n=-\sqrt{\epsilon \mu}$ [12]. It follows from the Maxwell's curl equations that the phase velocity of light wave propagating inside this medium is pointed opposite to the direction of energy flow, that is, the Poynting vector and wave vector of electromagnetic wave would be antiparallel, i.e., the vector $\mathbf{k}$, the electric field $\mathbf{E}$ and the magnetic field $\mathbf{H}$ form a left-handed system; thus Veselago referred to such materials as "left-handed" media, and correspondingly, the ordinary medium in which $\mathbf{k}, \mathbf{E}$ and $\mathbf{H}$ form a right-handed system may be termed the "right-handed" one. Other authors call this class of materials "negative-index media (NIM)" [13], "backward media (BWM)" [7], "double negative media (DNM)" [4] and Veselago's media. There exist a number of peculiar electromagnetic and optical properties, for instance, many dramatically different propagation characteristics stem from the sign change of the optical refractive index and phase velocity, including reversal of both the Doppler shift and Cherenkov radiation, anomalous refraction, amplification of evanescent waves [8], unusual photon tunneling [9], modified spontaneous emission rates and even reversals of radiation pressure to radiation

\footnotetext{
${ }^{1}$ Note that, in the literature, some authors mentioned the wrong year when Veselago suggested the left-handed media. They claimed that Veselago proposed or introduced the concept of left-handed media in 1968 or 1964. On the contrary, the true history is as follows: Veselago's excellent paper was first published in Russian in July, 1967 [Usp. Fiz. Nauk 92, 517-526 (1967)]. This original paper was translated into English by W.H. Furry and published again in 1968 in the journal of Sov. Phys. Usp. [12]. Unfortunately, Furry stated erroneously in his English translation that the original version of Veselago' work was first published in 1964.
} 
tension [2]. In experiments, this artificial negative electric permittivity media may be obtained by using the array of long metallic wires (ALMWs) [14], which simulates the plasma behavior at microwave frequencies, and the artificial negative magnetic permeability media may be built up by using small resonant metallic particles, e.g., the split ring resonators (SRRs), with very high magnetic polarizability [15-17]. A combination of the two structures yields a left-handed medium. Recently, Shelby et al. reported their first experimental realization of this artificial composite medium, the permittivity and permeability of which have negative real parts [3]. One of the potential applications of negative refractive index materials is to fabricate the so-called "superlenses" (perfect lenses): specifically, a slab of such materials may has the power to focus all Fourier components of a 2D image, even those that do not propagate in a radiative manner $[8,18]$.

A number of novel electromagnetic and optical properties in left-handed media result from the negative index of refraction. But what is its physical meanings? Although in the literature many researchers have investigated the negative refractive index of left-handed media by a variety of means of applied electromagnetism, classical optics, materials science as well as condensed matter physics $[1-6,10]$, less attention than it deserves is paid to the physical meanings of negative refractive index. In this Letter we will study this fundamental problem from the purely physical point of view.

Before we consider the negative refractive index of left-handed media, we first deal with the "optical refractive index" problem of de Broglie wave, the results of which will be helpful in discussing the former problem.

For the case of de Broglie particle moving in a force field, we can also consider its "optical refractive index" $n$. According to the Einstein- de Broglie relation, the dispersion relation of the de Broglie particle with rest mass $m_{0}$ in a scalar potential field $V(\mathbf{x})$ agrees with

$$
(\omega-\phi)^{2}=k^{2} c^{2}+\frac{m_{0}^{2} c^{4}}{\hbar^{2}}
$$

where $\phi$ is defined to be $\phi=\frac{V}{\hbar}$, and $k, \hbar$ and $c$ stand for the wave vector, Plank constant and speed of light in a vacuum, respectively. It follows from Eq.(1) that 


$$
\omega^{2}\left[1-\frac{m_{0}^{2} c^{4}}{\hbar^{2} \omega^{2}}-2 \frac{\phi}{\omega}+\left(\frac{\phi}{\omega}\right)^{2}\right]=k^{2} c^{2},
$$

which yields

$$
\frac{\omega^{2}}{k^{2}}=\frac{c^{2}}{1-\frac{m_{0}^{2} c^{4}}{\hbar^{2} \omega^{2}}-2 \frac{\phi}{\omega}+\left(\frac{\phi}{\omega}\right)^{2}} .
$$

Compared Eq.(3) with the dispersion relation $\frac{\omega^{2}}{k^{2}}=\frac{c^{2}}{n^{2}}$, one can arrive at

$$
n^{2}=1-\frac{m_{0}^{2} c^{4}}{\hbar^{2} \omega^{2}}-2 \frac{\phi}{\omega}+\left(\frac{\phi}{\omega}\right)^{2},
$$

which is the square of "optical refractive index" of de Broglie wave in the presence of a potential field $\phi$.

It is of physical interest to discuss the Fermat's principle of de Broglie wave in a potential field $V$. One can readily verify that the variation $\delta \int c n^{2} \mathrm{~d} t=0$ can serve as the mathematical expression for the Fermat's principle of de Broglie particle (for the derivation of this expression, the readers may be referred to the Appendix). If, for example, by using the weak-field and low-motion approximation where $\left|\frac{\phi}{\omega}\right| \ll 1, \frac{v^{2}}{c^{2}} \ll 1$ and $\left(\frac{\phi}{\omega}\right)^{2}$ in Eq.(4) can therefore be ignored, $1-\frac{m_{0}^{2} c^{4}}{\hbar^{2} \omega^{2}} \simeq \frac{v^{2}}{c^{2}}$, then $n^{2}$ is approximately equal to

$$
n^{2} \simeq \frac{2}{m c^{2}}\left(\frac{1}{2} m v^{2}-V\right)
$$

Thus in the non-relativistic case, Fermat's principle, $\delta \int c n^{2} \mathrm{~d} t=0$, is equivalent to the following well-known action principle $\delta \int L(\mathbf{x}, \mathbf{v}) \mathrm{d} t=0$ (differing only by a constant coefficient $\left.\frac{2}{m c^{2}}\right)$, where the function $L(\mathbf{x}, \mathbf{v})=\frac{1}{2} m v^{2}-V(\mathbf{x})$ denotes the Lagrangian of massive particle in a potential field $V(\mathbf{x})$. Hence the above formulation for the "optical refractive index" of the de Broglie particle inside a potential field is said to be self-consistent.

According to Eq.(4), there are two square roots (i.e., positive and negative roots, $n_{+}$, $n_{-}$) for the refractive index of de Broglie wave. Indeed, it is verified that the particle and its antiparticle possess these two square roots, respectively. If the refractive index of particle is positive, then that of its antiparticle will acquire a minus sign, which may be seen by utilizing the charge conjugation transformation: $\omega \rightarrow-\omega, \phi \rightarrow-\phi$. If, for example, we consider the 
refractive index of electron and positron by regarding the positron as the negative energy solution to Dirac's equation, then we can classify the solutions of Dirac's equation into the following four cases:

(i) $\quad E=E_{+}, \quad h=\hbar k, \quad n=n_{+}$;

(ii) $\quad E=E_{+}, \quad h=-\hbar k, \quad n=n_{+}$;

(iii) $\quad E=E_{-}, \quad h=\hbar k, \quad n=n_{-}$;

(iv) $\quad E=E_{-}, \quad h=-\hbar k, \quad n=n_{-}$,

where $h, E_{+}$and $E_{-}$respectively represent the helicity, positive and negative energy corresponding to the electron and positron. It is apparent that the relationship between the cases (i) and (iv) is just the charge conjugation transformation. This transformation also relates the case (ii) to (iii).

Compare the case of wave propagation in left-handed media with the results presented above, we can conclude that the negative optical refractive index in left-handed media corresponds to the antiparticles of photons (the so-called antiphotons). However, as is well known, there exist no antiphotons in free space. The theoretical reason for this is that the four-dimensional vector potentials $A_{\mu}$ with $\mu=0,1,2,3$ are always taking the real numbers. But in a dispersive and absorptive medium, as an effective medium theory, the vector potentials $A_{\mu}$ of which may probably take the complex numbers. Such complex vector field theory has been considered previously [19]. Here the Lagrangian density is written $\mathcal{L}=-\frac{1}{2} F_{\mu \nu}^{*} F_{\mu \nu}$, where the electromagnetic field tensors $F_{\mu \nu}=\partial_{\mu} A_{\nu}-\partial_{\nu} A_{\mu}, F_{\mu \nu}^{*}=\partial_{\mu} A_{\nu}^{*}-\partial_{\nu} A_{\mu}^{*}$ with $A_{\mu}^{*}$ being the complex conjugation of $A_{\mu}$. The complex four-dimensional vector potentials $A_{\mu}$ and $A_{\mu}^{*}$ describe the propagating behavior of both photons and antiphotons.

Now a problem left to us is that does the complex vector field theory apply well in investigating the light propagation inside the negative refractive index medium. Detailed analysis will show that this theory is truly applicable to the consideration of optical index of refraction and wave propagation in left-handed media. This will be proved in what follows by using another mathematical form 


$$
\frac{i}{c_{n}} \frac{\partial}{\partial t} \mathbf{M}=\nabla \times \mathbf{M}, \quad \nabla \cdot \mathbf{M}=0
$$

for the Maxwellian equations, where $\mathbf{M}=\sqrt{\epsilon} \mathbf{E}+i \sqrt{\mu} \mathbf{H}, c_{n}=1 / \sqrt{\epsilon \mu}, \epsilon$ and $\mu$ are absolute dielectric constant and magnetic conductivity of the medium, respectively. It can be easily verified that the Maxwellian equations can be rewritten as Eq.(6).

If $\epsilon>0$ and $\mu>0$ are assumed, then the light propagation inside the isotropic linear right-handed medium (regular medium) is governed by Eq.(6). For the time-harmonic electromagnetic wave in the right-handed medium, Eq.(6) can be rewritten

$$
i \frac{\omega}{c_{n}} \mathbf{M}=-\mathbf{k} \times \mathbf{M}
$$

with $\omega$ being the frequency of electromagnetic wave.

Now let us take the complex conjugation of the two sides of Eq.(7), and the result is of the form

$$
i \frac{\omega}{c_{n}} \mathbf{M}^{*}=\mathbf{k} \times \mathbf{M}^{*}
$$

where the field $\mathbf{M}^{*}$ is expressed in terms of both electric and magnetic fields, $\mathbf{E}^{*}$ and $\mathbf{H}^{*}$, i.e., $\mathbf{M}^{*}=\sqrt{\epsilon} \mathbf{E}^{*}-i \sqrt{\mu} \mathbf{H}^{*}$. Thus, we have

$$
i \frac{\omega}{c_{n}}\left[\sqrt{\epsilon} \mathbf{E}^{*}-i \sqrt{\mu} \mathbf{H}^{*}\right]=\mathbf{k} \times\left[\sqrt{\epsilon} \mathbf{E}^{*}-i \sqrt{\mu} \mathbf{H}^{*}\right] .
$$

Multiplying the two sides of Eq.(9) by the imaginary unit $i$, one can arrive at

$$
i \frac{\omega}{c_{n}}\left[\sqrt{-\epsilon} \mathbf{E}^{*}-i \sqrt{-\mu} \mathbf{H}^{*}\right]=\mathbf{k} \times\left[\sqrt{-\epsilon} \mathbf{E}^{*}-i \sqrt{-\mu} \mathbf{H}^{*}\right]
$$

where the magnitude of $c_{n}$ (i.e., $1 / \sqrt{\epsilon \mu}$ ) does not change but it can be rewritten as a new form $c_{n}=1 / \sqrt{(-\epsilon)(-\mu)}$. According to Eq.(10), one can obtain the following relations

$$
\mathbf{k} \times \mathbf{E}^{*}=\omega(-\mu) \mathbf{H}^{*}, \quad \mathbf{k} \times \mathbf{H}^{*}=-\omega(-\epsilon) \mathbf{E}^{*} .
$$

Note that here $\epsilon>0, \mu>0$. Thus it follows from Eq.(11) that the wave vector $\mathbf{k}$, the electric field $\mathbf{E}^{*}$ and the magnetic field $\mathbf{H}^{*}$ of the electromagnetic wave in this medium, 
through which the fields $\mathbf{E}^{*}$ and $\mathbf{H}^{*}$ propagate, form a left-handed system. This, therefore, means that here the Poynting vector $\mathbf{S}$ is pointed opposite to the direction of the wave vector of electromagnetic wave. For this reason, it is expected that the reversal of wave vector, instantaneous helicity inversion [20] and anomalous refraction will take place when an incident lightwave from the right-handed medium travels to the interfaces between leftand right- handed media. Since the direction of phase velocity and energy flow of lightwave propagating in the left-handed medium would be antiparallel, the change of wave vector $\mathbf{k}$ will truly occur during the light propagation through the interfaces. The inversion of $\mathbf{k}$ means that its magnitude acquires a minus sign, namely, the optical index of refraction $n$ becomes negative since the magnitude of $\mathbf{k}$ is defined to be $k=n \omega / c$. In view of the above discussion, it is concluded that Eq.(10) governs the propagation of time-harmonic planar electromagnetic wave, where the electromagnetic fields are described by $\mathbf{E}^{*}$ and $\mathbf{H}^{*}$.

Thus by making use of the complex vector field theory we obtain a formulation which can treat the wave propagation in both right- and left- handed media. One of the advantages of the present formulation is that the minus sign of negative refractive index can be placed into the theoretical calculations by using the above unified approach rather than by hand. Such unified approach will be useful in dealing with the electromagnetic wave propagation and photonic band gap structures in the so-called left-handed medium photonic crystals, which are artificial materials patterned with a periodicity in negative and positive index of refraction.

To summarize, we study the "optical refractive index" of de Broglie wave and reveal the physical meanings of negative refractive index in left-handed media. It is shown that the electromagnetic wave propagation in negative refractive index media behaves like that of antiphotons, which requires that we should take into account the complex vector field theory. This will provide us with a unified formulation to treat the wave propagation in both left- and right- handed media.

Acknowledgements This project was supported by the National Natural Science Founda- 
tion of China under the project No. 90101024. The author is grateful to S.L. He for useful discussion on the wave propagation in left-handed media.

\section{Appendix}

In this Appendix, we will discuss the Fermat's principle of de Broglie wave. First we consider the relativistic free-particle case where the Einstein-de Broglie relation $(\hbar k)^{2} c^{2}=$ $(\hbar \omega)^{2}-m_{0}^{2} c^{4}$ is utilized. By applying the dispersion relation $\frac{\omega^{2}}{k^{2}}=\frac{c^{2}}{n^{2}}$ to the Einstein-de Broglie relation, one can arrive at

$$
\frac{m_{0}^{2} c^{4}}{\hbar^{2}}=\left(1-n^{2}\right) \omega^{2}
$$

and consequently derive

$$
\hbar \omega=\frac{m_{0} c^{2}}{\sqrt{1-n^{2}}}
$$

Compared Eq.(A2) with the relativistic energy-velocity formula (with $v$ being the particle velocity)

$$
\hbar \omega=\frac{m_{0} c^{2}}{\sqrt{1-\left(\frac{v}{c}\right)^{2}}},
$$

we obtain $n=\frac{v}{c}$. According to the original expression for the Fermat's principle, i.e., $\delta \int n \mathrm{~d} l=0$, where $n \mathrm{~d} l$ is the "optical" path element with $\mathrm{d} l=v \mathrm{~d} t=n c \mathrm{~d} t$, the Fermat's principle for the de Broglie particle can be rewritten

$$
\delta \int \frac{v}{c} v \mathrm{~d} t=0 \quad \text { or } \quad \delta \int c n^{2} \mathrm{~d} t=0
$$

In order to consider the wave propagation of the Broglie particle in a potential field, we assume that the latter formula (i.e., $\left.\delta \int c n^{2} \mathrm{~d} t=0\right)$ in Eq.(A4) is still valid and would also apply very well in this case. It follows from Eq.(5)that this assumption truly holds at least for the non-relativistic case. 


\section{REFERENCES}

[1] D.R. Smith, W.J. Padilla, D.C. Vieret al., Phys. Rev. Lett. 84 (2000) 4184.

[2] V.V. Klimov, Opt. Comm. 211 (2002) 183.

[3] R.A. Shelby, D.R. Smith, and S. Schultz, Science 292 (2001) 77.

[4] R.W. Ziolkowski, Phys. Rev. E 64 (2001) 056625.

[5] J.A. Kong, B.L. Wu, and Y. Zhang, Appl. Phys. Lett. 80 (2002) 2084.

[6] N. Garcia and M. Nieto-Vesperinas, Opt. Lett. 27 (2002) 885.

[7] I.V. Lindell, S.A. Tretyakov, K.I.Nikoskinen, S. Ilvonen, Helsinki Uni. Technol., 366, ser. Electromagn. Lab. Rep. Ser., Apr. 2001.

[8] J.B. Pendry, Phys. Rev. Lett. 85 (2001) 3966.

[9] Z.M. Zhang, C.J. Fu, Appl. Phys. Lett. 80 (2002) 1097.

[10] J.Q. Shen, Phys. Scr. 68 (2003) 87.

[11] J.Q. Shen, Y. Jin, L. Chen, arXiv: cond-mat/0305457 (2003).

[12] V.G. Veselago, Sov. Phys. Usp. 10 (1968) 509.

[13] J. Gerardin and A. Lakhtakia, Phys. Lett. A 301 (2002) 377.

[14] J.B. Pendry, A.J. Holden, D.J. Robbins, and W.J. Stewart, J. Phys. Condens. Matter $10(1998) 4785$.

[15] J.B. Pendry, A.J. Holden, W.J. Stewart, and I. Youngs, Phys. Rev. Lett. 76 (1996) 4773.

[16] J.B. Pendry, A.J. Holden, D.J. Robbins, and W.J. Stewart, IEEE Trans. Microwave Theory Tech. 47 (1999) 2075.

[17] S.I. Maslovski, S.A. Tretyakov, and P.A. Belov, Inc. Microwave Opt. Tech. Lett. 35 
(2001) 47.

[18] G.W. t’ Hooft, Phys. Rev. Lett. 87 (2001) 249701.

[19] D. Lurié, Particles and Fields, Wiley, New York, 1968, Chap. 2.

[20] J.Q. Shen, L.H. Ma, Phys. Lett. A 308 (2003) 355. 